\title{
Characteristics of incomplete endobronchial ultrasound-guided transbronchial needle aspiration cases
}

\author{
Hidenao Kayawake ${ }^{1}$, Toyofumi F. Chen-Yoshikawa ${ }^{2}$, Satona Takana ${ }^{1}$, Yoshito Yamada ${ }^{1}$, Yojiro Yutaka ${ }^{1}$, \\ Daisuke Nakajima ${ }^{1}$, Masatsugu Hamaji ${ }^{1}$, Toshi Menju ${ }^{1}$, Akihiro Ohsumi ${ }^{1}$, Hiroshi Date ${ }^{1}$ \\ ${ }^{1}$ Department of Thoracic Surgery, Kyoto University, Kyoto, Japan; ${ }^{2}$ Department of Thoracic Surgery, Nagoya University Graduate School of \\ Medicine, Nagoya, Japan \\ Contributions: (I) Conception and design: H Kayawake, TF Chen-Yoshikawa, H Date; (II) Administrative support: None; (III) Provision of study \\ materials or patients: None; (IV) Collection and assembly of data: H Kayawake, TF Chen-Yoshikawa; (V) Data analysis and interpretation: H \\ Kayawake, TF Chen-Yoshikawa; (VI) Manuscript writing: All authors; (VII) Final approval of manuscript: All authors. \\ Correspondence to: Toyofumi F. Chen-Yoshikawa, MD, PhD. Department of Thoracic Surgery, Nagoya University Graduate School of Medicine, 65 \\ Tsurumai-cho, Showa-ku, Nagoya 466-8550, Japan. Email: fengshic@kuhp.kyoto-u.ac.jp.
}

Backgrounds: The number of endobronchial ultrasound-guided transbronchial needle aspiration (EBUSTBNA) cases is increasing due to its less-invasiveness and usefulness. However, there are several unresolved issues, including the existence of incomplete cases and complications. This study aimed to investigate the frequency and diagnostic management of incomplete EBUS-TBNA cases.

Methods: Between July 2009 and December 2017, 424 patients underwent EBUS-TBNA for the diagnosis of suspected malignancy. Among them, we retrospectively reviewed the frequency, characteristics and diagnostic managements of incomplete cases of EBUS-TBNA.

Results: EBUS-TBNA was not completed in 16 patients (3.8\%), all of whom underwent EBUS-TBNA cases under conscious sedation. The factors for incompleteness of EBUS-TBNA under conscious sedation were divided into two groups: impossibility to perform EBUS-TBNA under conscious sedation $(\mathrm{n}=8)$ and impossibility to perform safe needle biopsy $(n=8)$. The former factor consisted of strong coughing reflex and insufficient sedation $(n=5)$, frequent desaturation during EBUS-TBNA $(n=2)$, and allergy to lidocaine $(n=1)$, while the latter factor consisted of anatomical reasons $(n=4)$, abundant blood flow in the target lesion $(n=3)$, and invisibility of the target lesion due to airway deformation $(n=1)$. Eventually, 10 out of 16 cases were histologically diagnosed as having malignancy by a surgical approach $(\mathrm{n}=5)$ and EBUS-TBNA under general anesthesia $(\mathrm{n}=5)$.

Conclusions: Although the number was small, we did note some incomplete cases of EBUS-TBNA under conscious sedation. In incomplete cases under conscious sedation, EBUS-TBNA under general anesthesia and other surgical approaches can be considered as additional options.

Keywords: Endobronchial ultrasound-guided transbronchial needle aspiration (EBUS-TBNA); conscious sedation; general anesthesia; incomplete cases

Submitted Sep 28, 2019. Accepted for publication Nov 26, 2019.

doi: $10.21037 /$ jtd.2019.12.133

View this article at: http://dx.doi.org/10.21037/jtd.2019.12.133

\section{Introduction}

Endobronchial ultrasound-guided transbronchial needle aspiration (EBUS-TBNA) is widely used to diagnose enlarged thoracic lymph nodes (1-12). Because of the advances in imaging modalities, the accurate diagnosis of thoracic lymph nodes has become more important to provide appropriate treatment to patients with lung cancer (13).

In general, EBUS-TBNA under general anesthesia seems to be safer and more effective than that under 


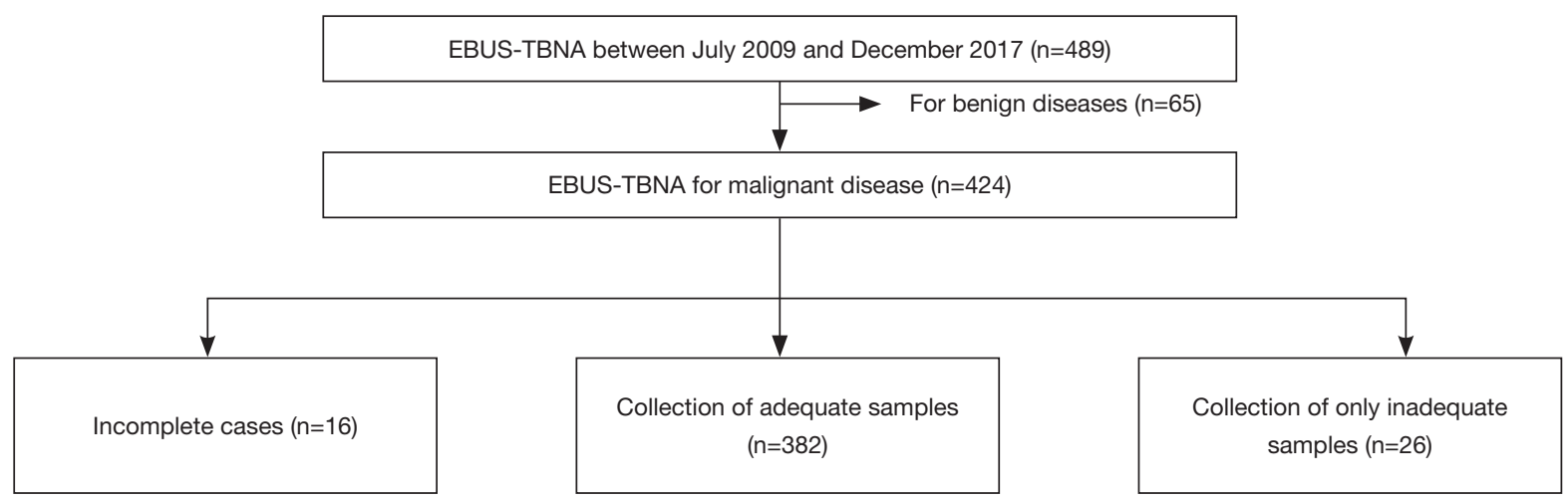

Figure 1 Between July 2009 and December 2017, 489 patients underwent EBUS-TBNA. Among them, this study included 424 patients undergoing EBUS-TBNA for the diagnoses of suspected malignancy. The number of incomplete cases was 16, and the number of cases with only inadequate sample collected was 26. EBUS-TBNA, endobronchial ultrasound-guided transbronchial needle aspiration.

conscious sedation. In some countries, such as Japan, most EBUS-TBNAs are performed under conscious sedation $(14,15)$. Most EBUS-TBNAs are completed successfully and few incomplete cases are encountered in daily practice. In this study, we reviewed EBUS-TBNA cases performed to diagnose suspected malignancy and investigated the frequency and diagnostic management of incomplete EBUS-TBNA cases.

\section{Methods}

Between July 2009 and December 2017, 489 EBUS-TBNAs were performed at Kyoto University Hospital. Some patients underwent EBUS-TBNA for benign diseases, but most patients underwent EBUS-TBNA for the diagnosis of malignant diseases. In this study, we reviewed 424 cases of EBUS-TBNA performed for the diagnoses of suspected malignancy (Figure 1).

In our institution, EBUS-TBNA is usually performed under conscious sedation with intravenous midazolam and local anesthesia with lidocaine. The patients were observed and local anesthesia was performed by bronchoscopy, after which EBUS-TBNA was performed. For EBUS-TBNA, a conventional bronchoscope (BF-Type 260; Olympus, Tokyo, Japan), a convex probe ultrasound bronchoscope (BF-UC260OF-OL8, BF-UC260FW; Olympus), and 21and 22-gauge needles (ViziShot ${ }^{\circledR}$, NA-201SX-4021 and NA201SX-4022; Olympus) were used. Selective biopsies of the preoperatively determined target lesions were performed in most cases. In cases of general anesthesia, the endoscopic procedures were performed under general anesthesia with intravenous propofol while the patients were intubated.
We defined incomplete cases as those in which the selected target lesions could not be punctured.

First, we investigated the frequency of incomplete EBUS-TBNA cases among the 424 cases. Then, we retrospectively reviewed the characteristics and diagnostic management of the incomplete cases of EBUS-TBNA. This study was approved by the Kyoto University Institutional Review Board (R1806). The requirement for informed consent from each patient was waived because of the retrospective nature of the study.

All statistical analysis was performed with EZR software (Saitama Medical Center, Jichi Medical University, Saitama, Japan), which is a graphical user interface for $\mathrm{R}$ (The R Foundation for Statistical Computing, Vienna, Austria) (16). All values are expressed as means \pm standard deviation or medians with range. Sensitivity, specificity, positive predictive value, and negative predictive value were calculated using the standard definitions.

\section{Results}

\section{Patient characteristics and the frequency of incomplete cases}

Altogether, 424 patients underwent EBUS-TBNA for diagnosis of suspected malignancy. The median age of the 424 patients was 68 years (range, 19-88 years). There were 303 male $(71.5 \%)$ and 121 female patients (28.5\%). Among the 424 patients, 415 patients underwent EBUSTBNA under conscious sedation and 9 patients underwent EBUS-TBNA under general anesthesia. The reasons for general anesthesia in these 9 cases were as follows: EBUS- 
Table 1 Patient characteristics, target lesions of EBUS-TBNA, and the number of punctures

\begin{tabular}{lc}
\hline Variables & Median [range] or number (\%) \\
\hline Age (years) & $68[19-88]$ \\
Sex & \\
Male & $303(71.5)$ \\
Female & $121(28.5)$ \\
Target lesion & \\
Mediastinal lesion & $306(72.2)$ \\
Hilar lesion & $68(16.0)$ \\
Both mediastinal and hilar & $50(11.8)$ \\
lesion & $2[0-9]$ \\
Number of punctures of \\
target lesion
\end{tabular}

EBUS-TBNA, endobronchial ultrasound-guided transbronchial needle aspiration.

Table 2 Factors associated with incomplete EBUS-TBNA cases divided into two types: impossibility to perform EBUS-TBNA under conscious sedation and impossibility to perform safe needle biopsy

\begin{tabular}{lc}
\hline Factors for incompleteness of EBUS-TBNA & Number (\%) \\
\hline $\begin{array}{l}\text { Impossibility to perform EBUS-TBNA under } \\
\text { conscious sedation }\end{array}$ & $5(62.5)$ \\
& $\begin{array}{l}\text { Due to strong cough reflex and insufficient } \\
\text { sedation }\end{array}$ \\
Due to frequent desaturation during EBUS- & $2(25.0)$ \\
TBNA & $1(12.5)$ \\
Due to allergy of lidocaine & \\
Impossibility to perform safe needle biopsy \\
$\begin{array}{l}\text { Due to the anatomical reasons } \\
\text { Due to abundant blood flow in target lesion }\end{array}$ \\
$\begin{array}{l}\text { Due to invisibility of target lesion due to airway } \\
\text { deformation }\end{array}$ & $1(12.5)$ \\
\hline
\end{tabular}

EBUS-TBNA, endobronchial ultrasound-guided transbronchial needle aspiration.

TBNA or bronchoscopy could not be completed because of a strong coughing reflex, frequent desaturation, or allergy to lidocaine $(n=7)$; mediastinoscopy was planned simultaneously when a definitive diagnosis could not be obtained via EBUS-TBNA $(n=1)$, and EBUS-TBNA under general anesthesia was recommended because of the suspected coexistence of pheochromocytoma $(\mathrm{n}=1)$.

In 306 patients $(72.2 \%)$, the target lesions of EBUSTBNA were mediastinal lesions and in 68 patients $(16.0 \%)$, they were hilar lesions, whereas in 50 patients $(11.8 \%)$, they were both hilar and mediastinal lesions. The median number of needle passes was 2 (range, 0-9; Table 1). EBUSTBNA procedures for mediastinal lesions were mainly performed as biopsies to diagnose the cause of mediastinal lymph node enlargement in patients with lung cancer or other malignancies and in cases with suspected mediastinal tumors. EBUS-TBNA procedures for hilar lesions were mainly performed as biopsies for the diagnosis of enlarged hilar lymph nodes and central lung lesions. Most of these biopsies were performed for the histological diagnosis of new hilar lesions (lymph nodes or central lung lesions) in patients with a history of treatment for previous malignancy. EBUS-TBNA procedures for both mediastinal and hilar lesions were mainly performed for the staging of lung cancers or malignant pleural mesotheliomas and to determine treatment strategies $(n=41)$. Other reasons for performing EBUS-TBNA of both lesions included establishing a histological diagnosis of new lesions in patients with a history of previous treatment for malignancy $(\mathrm{n}=5)$ and performing a biopsy of the suspected malignant lymphoma $(\mathrm{n}=4)$.

Among the 424 cases, EBUS-TBNA was not completed in 16 cases (3.8\%). All the 16 incomplete cases underwent EBUS-TBNA under conscious sedation, while the procedure was completed in all 9 patients undergoing EBUS-TBNA under general anesthesia.

\section{Factors associated with incomplete cases and final pathological diagnosis of malignancy among such cases}

The factors associated with the incomplete cases of EBUSTBNA could be divided into two groups: impossibility to perform EBUS-TBNA under conscious sedation $(\mathrm{n}=8)$ and impossibility to perform safe needle biopsy $(n=8)$. The impossibility to perform EBUS-TBNA under conscious sedation further consisted of 3 types of factors: strong coughing reflex and insufficient sedation $(n=5)$, frequent desaturation during EBUS-TBNA $(n=2)$, and allergy to lidocaine $(n=1)$. The impossibility to perform safe needle biopsy also consisted of 3 types of factors: anatomical reasons $(n=4)$, abundant blood flow in the target lesion $(\mathrm{n}=3)$, and invisibility of the target lesion due to airway deformation ( $\mathrm{n}=1$; Table 2). The anatomical reasons meant that the target lesions were located adjacent to critical 

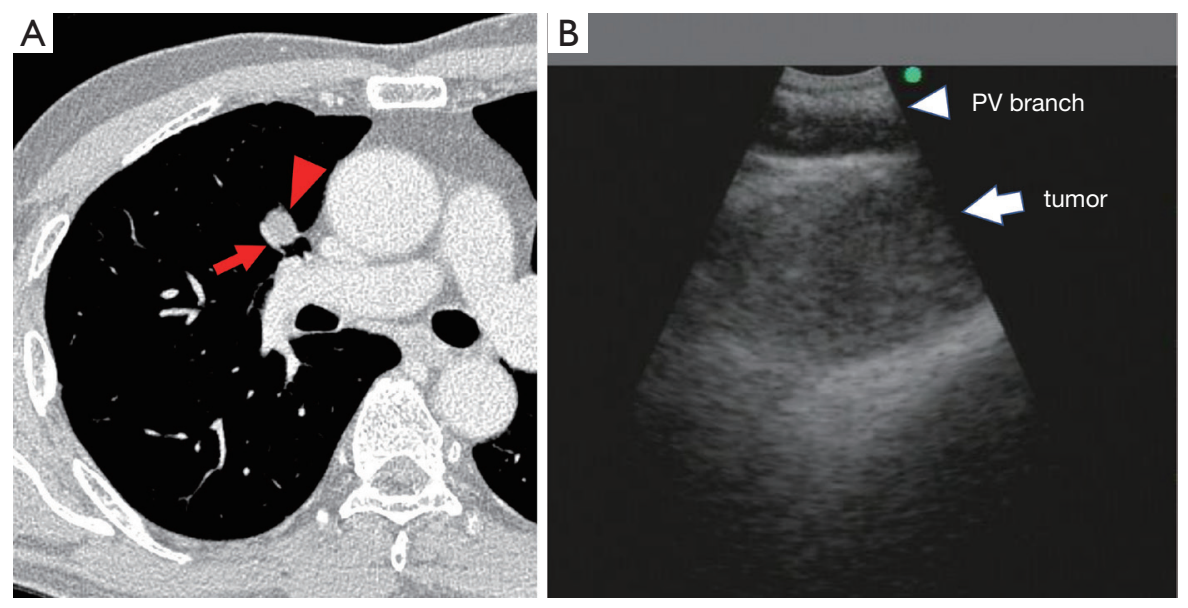

Figure 2 A typical case whose target lesion is located adjacent to the pulmonary venous branch. (A) Axial chest CT image of a case wherein the target lesion (arrow head) was located adjacent to the pulmonary arterial branch (arrow). (B) EBUS-TBNA scan of a patient whose tumor was adjacent to the pulmonary venous branch. The arrow head is showing the pulmonary venous branch and the arrow is showing the target tumor. EBUS-TBNA, endobronchial ultrasound-guided transbronchial needle aspiration; CT, computed tomography; PV, pulmonary vein.

vessels, such as branches of the pulmonary artery and vein (Figure 2).

Among the 16 incomplete cases, 10 cases were eventually pathologically diagnosed as having malignancy. Among the 8 incomplete cases due to the impossibility to perform EBUS-TBNA under conscious sedation, 5 cases were diagnosed by EBUS-TBNA under general anesthesia, and 1 case was diagnosed by curative surgery. On the other hand, among the 8 incomplete cases due to the impossibility to perform safe needle biopsy, 4 cases were diagnosed by surgical biopsy or curative surgery. Among the remaining 2 incomplete cases due to the impossibility to perform EBUSTBNA under conscious sedation, 1 case was diagnosed clinically by CT images as having malignancy, and the other case was diagnosed as not having malignancy by EBUSTBNA under general anesthesia. Conversely, among the remaining 4 incomplete cases due to the impossibility to perform safe needle biopsy, 2 cases were diagnosed clinically by CT images as having malignancy, and the remaining 2 cases were pathologically diagnosed as not having malignancy by curative surgery or surgical biopsy (Table 3). As a result, prolonged hospitalization was required in 2 patients for additional examinations and readmission due to additional examinations was required in 6 patients.

\section{Diagnostic accuracy and complications of EBUS-TBNA}

Among 408 complete EBUS-TBNA cases, adequate samples were collected in 382 cases. Among these 382 cases, the sensitivity, specificity, positive predictive value, and negative predictive values were calculated as $90.0 \%$, $100 \%, 100 \%$, and $76.0 \%$ (true positive in 261 , false positive in 0 , true negative in 92 , and false negative in 29 patients), respectively.

Of the 424 patients who underwent EBUS-TBNA, 4 experienced complications of EBUS-TBNA. These complications included mediastinitis $(\mathrm{n}=2)$, obstructive pneumonia $(\mathrm{n}=1)$, and airway obstruction requiring admission to the intensive care unit $(n=1)$.

\section{Discussion}

In this study, two important findings were noted. First, a few incomplete cases of EBUS-TBNA under conscious sedation were noted. Second, the factors for the incompleteness of EBUS-TBNA under conscious sedation were divided into two groups: impossibility to perform EBUS-TBNA under conscious sedation and impossibility to safely perform a needle biopsy. Consequently, for the diagnostic management of the incomplete cases, EBUS-TBNA under general anesthesia was often performed due to the former factor, while other surgical modalities were employed due to the latter factor.

In our institution, EBUS-TBNA was tentatively introduced in 2006 and has been performed in earnest since July 2009. As in many institutions in Japan, EBUS- 
Table 3 A summary of the 16 incomplete EBUS-TBNA cases

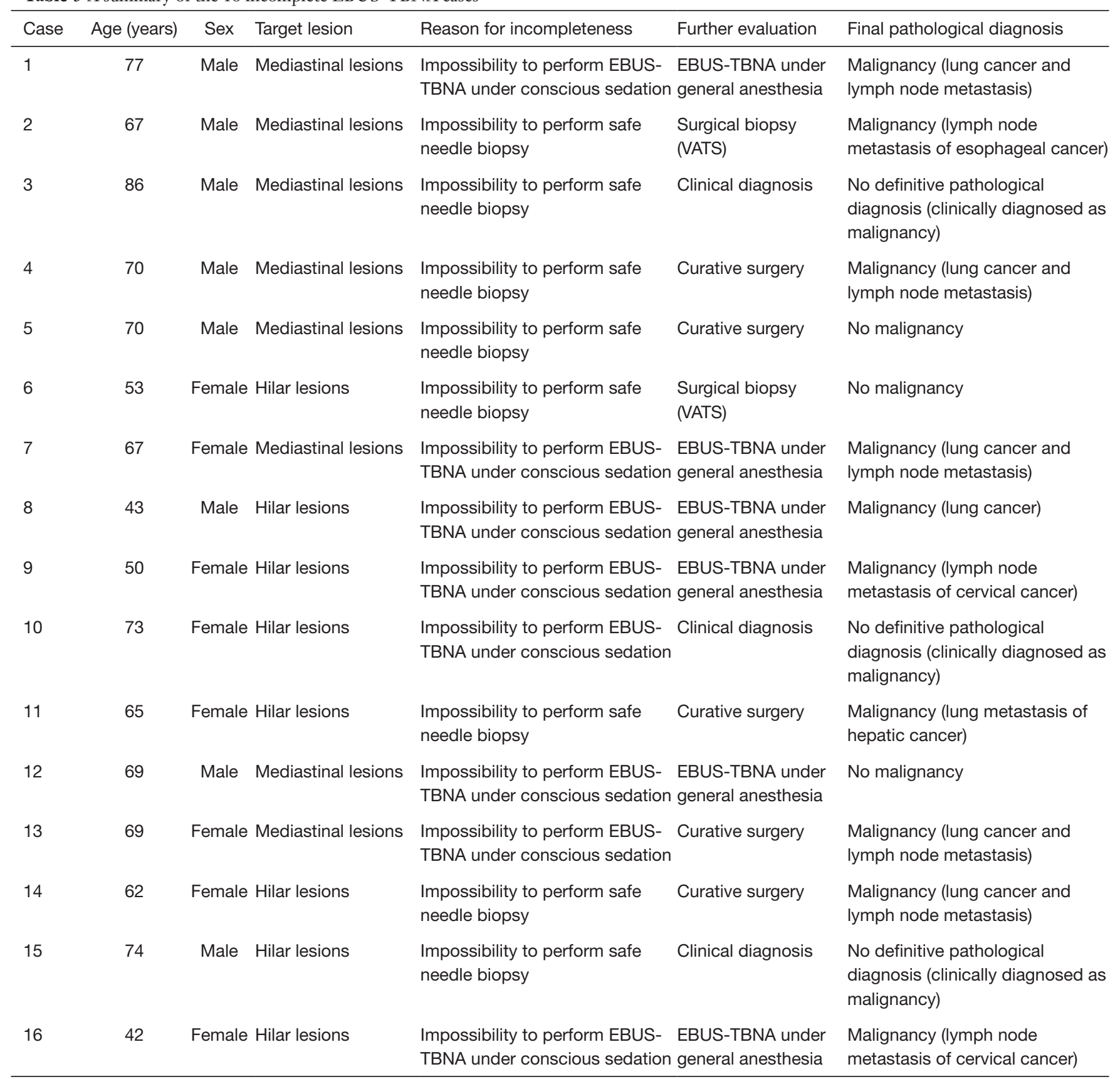

Among the 8 incomplete cases due to the impossibility to perform EBUS-TBNA under conscious sedation, 5 cases were diagnosed as having malignancy by EBUS-TBNA under general anesthesia, and 1 case was by curative surgery. Among the remaining 8 incomplete cases due to the impossibility to perform safe needle biopsy, 4 cases were diagnosed as having malignancy by surgical approach. EBUSTBNA, endobronchial ultrasound-guided transbronchial needle aspiration; VATS, video-assisted thoracoscopic surgery. 
TBNA is usually performed under conscious sedation at our hospital and selective biopsies of the supposed target lesions are usually performed $(14,17,18)$. The completion rate seemed to be satisfactory; however, since there were several incomplete cases, approaches to decrease the number of incomplete cases should be investigated in the future.

There are a few reports on the existence of incomplete cases of EBUS-TBNA (19); however, the details of these cases have not been reported. In our study, incomplete cases were found to be divided into two groups: cases in which EBUS-TBNA was impossible to perform under conscious sedation and cases wherein EBUS-TBNA was impossible with a safe needle biopsy. The former problem can be resolved by performing EBUS-TBNA under general anesthesia. Narcotic drugs were reported to possibly decrease the coughing reflex during EBUS-TBNA procedures under conscious sedation $(19,20)$. However, due to the necessity of the strict control of narcotic drugs, the Japanese guidelines for bronchoscopy do not recommend their use. In fact, narcotic drugs for EBUS-TBNA have never been used in our hospital to date. Safer and more effective sedation methods are needed to reduce the number of cases in which appropriate conscious sedation cannot be achieved.

In terms of the impossibility to safely perform needle biopsies, some factors might be considered before EBUSTBNA is initiated. We found that the most frequent factor correlated with the impossibility to safely perform needle biopsies was the location of the target lesions being adjacent to vessels. In these cases, as much effort as possible is made to determine an appropriate route to the target lesions that is sufficiently away from vessels. However, when such a suitable route cannot be determined, biopsies are performed in a transvascular manner. Transvascular approaches are usually avoided due to the risk of massive bleeding. Although, according to a recent report (21), these approaches can be safely performed, after considering the risks and benefits it would be better to avoid a transvascular approach if other approaches, such as surgical biopsy, are available since EBUS-TBNA is a diagnostic procedure and safety is most important.

In addition, abundant blood flow in the target lesion was noted as another factor correlated with the impossibility to safely perform needle biopsies. In some cases, EBUS-TBNA for target lesions with abundant blood flow can be safely performed and contribute to the differential diagnosis (17); however, in other cases, bleeding during EBUS-TBNA can occur. Therefore, close attention must be paid to lesions with abundant blood flow, and whether needle biopsy can be performed should be carefully considered.

We made efforts to reach a definitive diagnosis using other modalities, such as mediastinoscopy and videoassisted thoracoscopic surgery (VATS), in cases that were strongly suspected to have malignancy even though a definitive diagnosis using EBUS-TBNA was not possible. Some patients underwent curative resection directly after EBUS-TBNA. Thus, since the thoracic surgeon can employ multiple diagnostic approaches in addition to having a precise understanding of the mediastinal and thoracic anatomy, EBUS-TBNA performed by thoracic surgeons seems to be beneficial. Conversely, there have been some reports showing that the diagnostic accuracy of the combination of EBUS-TBNA and endoscopic transesophageal ultrasound-guided fine needle aspiration (EUS-FNA) was higher than that of EBUS-TBNA alone $(22,23)$. In fact, 3 patients in this study also underwent EUS-FNA on days other than the one on which EBUSTBNA was performed. However, these two examination approaches are not performed simultaneously in many institutions including our institution. Taken together, to achieve better diagnostic accuracy, we should select the appropriate diagnostic approach in a patient-specific manner.

This study has some limitations. First, although the number of patients included was relatively large, this was a retrospective, single institution, observational study. Second, we do not routinely perform lung cancer staging using EBUS-TBNA; however, selective biopsies are usually performed since EBUS-TBNA is mainly employed to determine treatment plans in our institution. Further, in cases where curative operations are performed after EBUS-TBNA, all the lymph nodes examined using EBUSTBNA are completely dissected. Third, the EBUS-TBNA operators vary in our institution; therefore, to maintain the level of diagnostic accuracy, both appropriate training and feedback are important.

\section{Conclusions}

Although most EBUS-TBNA procedures under conscious sedation can be completed, incomplete cases under conscious sedation do exist. Among the incomplete cases, EBUS-TBNA under general anesthesia and other diagnostic approaches, such as mediastinoscopy and VATS, can be performed to enhance the diagnostic accuracy, especially in cases that are strongly suspected of having 
malignancy.

\section{Acknowledgments}

An outline of this manuscript was presented at the International Association for the Study of Lung Cancer 19th World Conference on Lung Cancer in Toronto, Canada, 2018. The authors thank the pathologists, pulmonologists, medical engineers, and clinical laboratory technologists in Kyoto University Hospital for their support of EBUSTBNA.

Funding: None.

\section{Footnote}

Conflicts of Interest: M Hamaji and TF Chen-Yoshikawa serve as the unpaid editorial board members of fournal of Thoracic Disease from Oct 2018 to Sep 2020 and from Apr 2019 to Mar 2021 respectively. The other authors have no conflicts of interest to declare.

Ethical Statement: The authors are accountable for all aspects of the work in ensuring that questions related to the accuracy or integrity of any part of the work are appropriately investigated and resolved. This study was approved by the Kyoto University Institutional Review Board (R1806). The requirement for informed consent from each patient was waived because of the retrospective nature of the study.

Open Access Statement: This is an Open Access article distributed in accordance with the Creative Commons Attribution-NonCommercial-NoDerivs 4.0 International License (CC BY-NC-ND 4.0), which permits the noncommercial replication and distribution of the article with the strict proviso that no changes or edits are made and the original work is properly cited (including links to both the formal publication through the relevant DOI and the license). See: https://creativecommons.org/licenses/by-nc-nd/4.0/.

\section{References}

1. Yasufuku K, Chiyo M, Sekine Y, et al. Real-time Endobronchial Ultrasound-Guided Transbronchial Needle Aspiration of Mediastinal and Hilar Lymph Nodes. Chest 2004;126:122-8.

2. Yasufuku K, Chiyo M, Koh E, et al. Endobronchial ultrasound guided transbronchial needle aspiration for staging of lung cancer. Lung Cancer 2005;50:347-54.

3. Lee HS, Lee GK, Lee HS, et al. Real-time Endobronchial Ultrasound-Guided Transbronchial Needle Aspiration in Mediastinal Staging of Non-Small Cell Lung Cancer; How Many Aspirations Per Target Lymph Node Station? Chest 2008;134:368-74.

4. Herth FJF, Eberhardt R, Krasnik M, et al. Endobronchial Ultrasound-Guided Transbronchial Needle Aspiration of Lymph Nodes in the Radiologically and Positron Emission Tomography-Normal Mediastinum in Patients With Lung Cancer. Chest 2008;133:887-91.

5. Oki M, Saka H, Kitagawa C, et al. Prospective study of endobronchial ultrasound-guided transbronchial needle aspiration of lymph nodes versus transbronchial lung biopsy of lung tissue for diagnosis of sarcoidosis. J Thorac Cardiovasc Surg 2012;143:1324-9.

6. Chen F, Miyahara R, Sato T, et al. Usefulness of endobronchial ultrasound in patients with previously treated thoracic malignancy. Interact Cardiovasc Thorac Surg 2012;14:34-7.

7. Gupta D, Dadhwal DS, Agarwal R, et al. Endobronchial Ultrasound-Guided Transbronchial Needle Aspiration vs Conventional Transbronchial Needle Aspiration in the Diagnosis of Sarcoidosis. Chest 2014;146:547-56.

8. Yasufuku K, Pierre A, Darling G, et al. A prospective controlled trial of endobronchial ultrasound-guided transbronchial needle aspiration compared with mediastinoscopy for mediastinal lymph node staging of lung cancer. J Thorac Cardiovasc Surg 2011;142:1393400.e1.

9. Sanz-Santos J, Serra P, Torky M, et al. Systematic Compared With Targeted Staging With Endobronchial Ultrasound in Patients With Lung Cancer. Ann Thorac Surg 2018;106:398-403.

10. Hashimoto K, Daddi N, Giuliani M, et al. The role of endobronchial ultrasound-guided transbronchial needle aspiration in stereotactic body radiation therapy for nonsmall cell lung cancer. Lung Cancer 2018;123:1-6.

11. Vial MR, O'Connell OJ, Grosu HB, et al. Diagnostic performance of endobronchial ultrasound-guided mediastinal lymph node sampling in early stage nonsmall cell lung cancer: A prospective study. Respirology 2018;23:76-81.

12. Um SW, Kim HK, Jung SH, et al. Endobronchial ultrasound versus mediastinoscopy for mediastinal nodal staging of non-small-cell lung cancer. J Thorac Oncol 2015;10:331-7.

13. Silvestri GA, Gonzalez AV, Jantz MA, et al. Methods for 
Staging Non-small Cell Lung Cancer. Diagnosis and Management of Lung Cancer, 3rd ed: American College of Chest Physicians Evidence-Based Clinical Practice Guidelines. Chest 2013;143:e211S-50S.

14. Kayawake H, Chen-Yoshikawa TF, Oda H, et al. Complications of Endobronchial Ultrasound-Guided Transbronchial Needle Aspiration. Ann Thorac Surg 2017;104:e363-5.

15. Gochi F, Chen F, Aoyama A, et al. Mediastinal infectious complication after endobronchial ultrasound-guided transbronchial needle aspiration. Interact Cardiovasc Thorac Surg 2013;17:751-2.

16. Kanda Y. Investigation of the freely available easy-touse software 'EZR' for medical statistics. Bone Marrow Transplant 2013;48:452-8.

17. Chen F, Yoshizawa A, Okubo K, et al. Characteristic endobronchial ultrasound image of hemangiopericytoma/ solitary fibrous tumor. Interact Cardiovasc Thorac Surg 2010;11:331-2.

18. Chen F, Miyahara R, Sato T, et al. Usefulness of endobronchial ultrasound in patients with previously treated thoracic malignancy. Interact Cardiovasc Thorac Surg 2012;14:34-7.

Cite this article as: Kayawake H, Chen-Yoshikawa TF, Takana S, Yamada Y, Yutaka Y, Nakajima D, Hamaji M, Menju T, Ohsumi A, Date H. Characteristics of incomplete endobronchial ultrasound-guided transbronchial needle aspiration cases. J Thorac Dis 2020;12(3):573-580. doi: 10.21037/jtd.2019.12.133
19. Casal RF, Lazarus DR, Kuhl K, et al. Randomized trial of endobronchial ultrasound-guided transbronchial needle aspiration under general anesthesia versus moderate sedation. Am J Respir Crit Care Med 2015;191:796-803.

20. Jeyabalan A, Medford AR. Endobronchial ultrasoundguided transbronchial needle aspiration: patient satisfaction under light conscious sedation. Respiration 2014;88:244-50.

21. Kazakov J, Hegde P, Tahiri M, et al. Endobronchial and Endoscopic Ultrasound-Guided Transvascular Biopsy of Mediastinal, Hilar, and Lung Lesions. Ann Thorac Surg 2017;103:951-5.

22. Oki M, Saka H, Ando M, et al. Endoscopic ultrasoundguided fine needle aspiration and endobronchial ultrasound-guided transbronchial needle aspiration: Are two better than one in mediastinal staging of nonsmall cell lung cancer? J Thorac Cardiovasc Surg 2014;148:1169-77.

23. Lee KJ, Suh GY, Chung MP, et al. Combined endobronchial and transesophageal approach of an ultrasound bronchoscope for mediastinal staging of lung cancer. PLoS One 2014;9:e91893. 